\title{
Criminologie
}

\section{Récit de voyage à l'intérieur du Code criminel de 1892 où entre autres mention est faite des peines auxquelles ses transgresseurs s'exposent}

\section{Fernando Acosta}

Volume 28, numéro 1, 1995

L'histoire de l'enfermement et des populations pénales

URI : https://id.erudit.org/iderudit/017365ar

DOI : https://doi.org/10.7202/017365ar

Aller au sommaire du numéro

Éditeur(s)

Les Presses de l'Université de Montréal

ISSN

0316-0041 (imprimé)

1492-1367 (numérique)

Découvrir la revue

Citer cet article

Acosta, F. (1995). Récit de voyage à l'intérieur du Code criminel de 1892 où entre autres mention est faite des peines auxquelles ses transgresseurs s'exposent. Criminologie, 28(1), 81-96. https://doi.org/10.7202/017365ar

\section{Résumé de l'article}

This article introduces some preliminary results from an ongoing research project on the history of the Canadian Criminal Code. These results focus mainly on two important aspects of the first version, promulgated in 1892, of this Code : the structure which has ruled over its construction (and which remains overwhelmingly untouched to this day), and the place of emprisonment in the overall framework of the sentencing provisions of the Code. A short explanation of some of the difficulties surrounding the identification and counting of the incriminatory statements in the Code's text precedes the presentation of these results. By way of conclusion, the author identifies one research avenue to which a detailed analysis of the legal texts in the field of penal law seems to point. It refers to the movement of widening and narrowing of the regulation power of the State, particularly in the fields of (de)criminalization and (de)penalization. 
RÉCIT DE VOYAGE À L'INTÉRIEUR DU CODE CRIMINEL DE 1892

OÙ ENTRE AUTRES MENTION EST FAITE DES PEINES AUXQUELLES SES TRANSGRESSEURS S'EXPOSENT ${ }^{1}$

Fernando Acosta ${ }^{2}$

This article introduces some preliminary results from an ongoing research project on the history of the Canadian Criminal Code. These results focus mainly on two important aspects of the first version, promulgated in 1892, of this Code: the structure which has ruled over its construction (and which remains overwhelm. ingly untouched to this day), and the place of emprisonment in the overall framework of the sentencing provisions of the Code. A short explanation of some of the difficulties surrounding the identification and counting of the incriminatory statements in the Code's text precedes the presentation of these results. By way of conclusion, the author identifies one research avenue to which a detailed analysis of the legal texts in the field of penal law seems to point. It refers to the movement of widening and narrowing of the regulation power of the State, particularly in the fields of (de)criminalization and (de)penalization.

De combien de crimes pouvons-nous être coupables? Personne ne le sait, et pourtant nul n'est censé ignorer la loi. Ce paradoxe, sans doute l'un des plus déconcertants sur lequel repose l'entreprise pénale, acquiert dans le contexte particulier de notre droit pénal positif des contours encore plus affolants. Deux facteurs, entre autres, contribuent à cet état de choses : l'un externe, l'autre interne au Code. Il suffit de penser, pour le premier, à la multitude de lois sectorielles qui instituent des incriminations en marge du Code pour se rendre compte de la difficulté de connaître les dispositions de la loi pénale et, par conséquent, de s'y plier. En ce qui concerne le facteur interne - celui qui nous intéresse plus particulièrement dans cet article nous faisons allusion au fait que le Code criminel canadien n'est qu'en partie ce que son nom indique à première vue, soit un répertoire d'infractions, un recueil d'interdictions dont la transgression est frappée d'une peine. Construit avec des matériaux divers, le Code est aussi un catalogue

1. L'assise empirique de ce travail a été constituée dans le cadre d'une recherche sur l'histoire du Code criminel canadien, menee en collaboration avec Alvaro Pires et André Cellard, subventionnée par le Conseil de recherches en sciences humaines du Canada. Nous tenons à remercier notre assistante Chantal Collin-Dunn, étudiante de doctorat du Département d'histoire de l'Université d'Ottawa, de l'aide précieuse qu'eile a apportée aux différentes phases de la cueillette de données de ce projet.

2. Professeur, Département de criminologie de l'Université d'Ottawa, 1, Stewart, Ottawa, Ontario, KIN 6 N5. 
de règles de procédure et de preuve et, encore, de définitions, de règles d'exclusion d'imputabilité, de critères de sanction, de directives, d'instructions et nous en passons. Débusquer l'énoncé d'incrimination, dans les méandres de ce texte complexe, écrit dans une langue souvent impénétrable, constitue en réalité plus qu'un défi à l'imagination de chacun d'entre nous - c'est une tâche qui soulève des problèmes théoriques et méthodologiques dont on commence à peine à évaluer l'ampleur.

Mais en fait, à quel Code criminel faisons-nous référence ? À celui que l'on applique aujourd'hui, sur l'ensemble du territoire canadien, ou plutôt au Code que le titre de cet article annonce, le premier dont s'est doté le Canada suite à une longue trajectoire qui commence lors de la Conquête de la Nouvelle-France et se termine vingt-cinq ans après la Confédération? Y a-t-il des différences significatives entre ces deux textes qu'un siècle d'histoire sépare? Oui et non.

Comme on l'a déjà souvent mentionné, le Code actuel s'est débarrassé, du moins en partie, de cette tendance qui a si profondément marqué le Code de 1892 qui consistait à tout prévoir, tout montrer, tout dire (et parfois deux fois plutôt qu'une), tendance qui fera de lui un véritable monument d'hyperréalisme juridique. Mais la rupture la plus nette entre ces deux textes remonte en fait à 1955, moment où le common law sera partiellement banni du droit pénal canadien par l'interdiction d'y recourir dans le but de créer une infraction ${ }^{3}$. À toute règle au moins une exception, on laissera aux tribunaux le soin de préciser ce que peut être un outrage au tribunal, la seule infraction de common law qui subsiste de nos jours, malgré le fait que certaines de ces formes aient été codifiées 4.

Néanmoins, il est également de bonnes raisons de croire que ces différences sont plutôt formelles, ou dit autrement, qu'un code né sous l'inspiration du common law restera toujours profondément attaché à cette tradition. Cela est soutenable, à plus forte raison, lorsque l'on pense que c'est surtout là où le droit coutumier britannique s'est le plus illustrét que le droit pénal canadien éprouve le plus de difficultés à asseoir définitivement son autononle. Nous évoquons ici cet ensemble complexe, et souvent incompréhensible, de règles de procédure et de preuve qui, tout en encombrant déjà de manière considérable le texte du Code, se trouve en permanence soumis aux fluctuations des tendances jurisprudentielles. Un siècle d'histoire de notre Code n'a pas modifié fondamentalement cette réalité. Quoi qu'il en soit, l'argument sans doute le plus valable à l'appui de la

3. Cf. Commission de reforme du droit du Canada, Pour une codification du droit pénal, Ottawa, 1976, p. 31 .

4. Ibid., p. 31. Voir également Commission de réforme du droit du Canada, L'Outrage au tribunal, Ottawa, 1977.

5. Op. cit., note 3, p. 8. 
thèse de l'invariance du Code, cent ans après sa naissance, tient surtout à l'architecture de sa construction. Les nombreux changements dont ce texte a fait l'objet au cours de son existence (en 1906, 1927, 1955, 1970 et 1985, sans compter, bien entendu, les amendements qui s'ajoutent, année après année), affectant la forme et, dans certains cas, le fond de ses dispositions, l'ont très peu modifié ${ }^{6}$. À cette seule différence près que cette architecture est aujourd'hui bien moins cohérente et défendable qu'elle ne l'était à l'origine. Un retour au moule initial du Code qui nous gouverne aujourd'hui semble donc devoir s'imposer, d'autant plus qu'un tel exercice ne semble pas être fréquent dans la sociologie juridique qui se pratique actuellement au Québec.

En guise d'introduction, nous proposerons dans la première partie de ce texte un certain nombre de réflexions qui, en dépit de leur état fragmentaire, illustrent quelques-uns des problèmes théoriques et méthodologiques qu'une lecture du Code soulève. Cette toile de fond, dont on aperçoit déjà les contours à travers les questions évoquées ci-haut, nous semble essentielle à la compréhension des thèmes principaux de cet article. Dans la deuxième section du texte, nous allons examiner, à tour de rôle, la structure générale du Code, son dispositif de sanctions et, finalement, la place qu'y occupe la peine d'emprisonnement. L'état actuel de nos travaux de recherche nous oblige, pour l'instant, à traiter de ces questions sous un angle essentiellement descriptif.

\section{EST COUPABLE DE...}

L'affirmation «On ouvre un Code criminel pour y chercher des crimes" vaut sûrement, à celui qui ose tenir un tel propos, l'opprobre d'avoir énoncé une formidable banalité. Et pourtant, ce n'en est pas aussi simple. Les conduites incriminées ne sont pas aussi évidentes qu'on pourrait le croire, à première vue, à la lecture du texte du Code. Cela s'explique pour plusieurs raisons, et le fait que les énoncés d'incrimination se trouvent allègrement mélangés à des énoncés d'autre nature - comme il est évoqué plus haut — n'en est qu'une parmi bien d'autres. D'autres raisons, peut-être encore plus importantes, pointent plutôt vers la technique d'écriture du Code, ou, plus précisément, mettent en évidence l'absence d'un modèle invariable (et donc prévisible) de transmission du message dont il est porteur. Ainsi, les énoncés d'incrimination peuvent revêtir différentes formes ${ }^{7}$.

La forme la plus courante d'écriture des incriminations dans le Code, que tous reconnaissaient sans difficulté, est celle qui énonce sans ambages

6. Ibid., p. 32.

7. Cette affirmation vaut aussi bien pour les actes criminels que pour les infractions punissables par voie sommaire de culpabilité. 
la qualification pénale de l'acte et les conséquences qu'il entraîne : « Est coupable d'un acte criminel et passible d'un an d'emprisonnement... » C'est de toute évidence la forme la plus rassurante pour tous ceux qui se heurtent aux difficultés d'interprétation de ce texte et, particulièrement, le chercheur.

On incrimine, également, en se servant de la partie initiale de la formule rituelle ci-haut, mais sans énoncer aucune peine. La sanction, on la trouvera quelques pages plus loin, ou dans une autre partie du Code, ou... nulle part. Dans ce dernier cas, le cercle de l'incrimination ne se ferme qu'à l'aide d'une peine omnibus, généralement renvoyée à la toute fin du Code (en 1892, on l'a prévue à l'article 951, et elle était de sept ans d'emprisonnement, au maximum). Une façon plus insidieuse d'énoncer cette forme d'incrimination consiste à définir, dans un premier temps, un comportement (exemple classique : le meurtre) et, ensuite, à le criminaliser à l'aide du procédé courant décrit plus haut. Cette forme, en principe simple à décoder, peut prendre l'allure d'un véritable casse-tête lorsque l'énoncé d'incrimination ajoute (ou semble ajouter) des éléments nouveaux à l'énoncé de définition qui le précède. Parle-t-on encore de l'acte qui semblait déjà définitivement circonscrit par le texte préalable de définition ou d'un autre acte? Ou devrait-on plutôt attribuer aux éléments nouveaux la fonction d'exclure des moyens de défense ${ }^{8}$, utilisés en cour, pour échapper au cadre (événementiel, temporel, etc.) tracé par la définition? Si tel est le cas, pourquoi $n$ 'a-t-on pas prévu cette possibilité dans la définition elle-même? On pourrait multiplier ad nauseam de telles considérations, surtout lorsque l'on pense que d'autres combinaisons encore plus complexes de définitions, énoncés de sanctions et formes courantes d'incrimination sont également possibles, alourdissant ainsi considérablement la tâche de défrichement du Code. Il serait cependant trop fastidieux de les dénombrer ici.

Il n'est donc pas exagéré de prétendre que peu de textes posent d'obstacles aussi tenaces à la compréhension que ceux dont on se sert pour dire la loi pénale. Non pas qu'ils soient plus «difficiles » que d'autres, au sens que l'on attribue couramment à cette épithète, c'est qu'ils sont truffés de pièges. Cunsidérons à titre d'exemple le texte suivant.

Tout individu qui entreprend de faire une chose dont l'omission est ou peut être dangereuse pour la vie humaine, est légalement tenu de faire cette chose et est criminellement responsable des conséquences de son omission, si, sans excuse légitime, il ne remplit pas ce devoir. (C'est nous qui soulignons.)

8. Le common law est le fondement, encore aujourd'hui, pour un certain nombre de moyens de preuve non prévus dans le Code. Cf. G.C. Harper et al., Droit pénal canadien, Cowansville, Yvon Blais, 1989, pp. 31-32. 
C'est l'article 214 du Code criminel de 1892. S'agit-il d'une autre variante des formes d'incrimination? En absence de l'énoncé «Est coupable de...», on serait tenté de dire non. On pourrait alors y voir, par tâtonnements successifs, le procédé d'exclusion d'un moyen de défense dont on a parlé plus haut, ou simplement une règle d'interprétation en matière d'omissions. Ces solutions présentent cependant de sérieux problèmes. En effet, l'article 214 est le dernier d'une série de six inclus dans la Partie XVI du Code de 1892 qui traite des « devoirs tendant à la conservation de la vie ". Pour les trois premiers articles de la série $(209 \text { à } 211)^{9}$, le cercle de l'incrimination se ferme à l'aide de l'article 215 . Ils créent donc des crimes punissables en l'occurrence d'une peine maximale de trois ans. Pour les trois derniers (articles 212 à 214) ${ }^{10}$, par contre, on ne trouve aucune sanction spécifique, même si leur mode de construction est en tous points identique à celui des trois articles qui les précèdent. Au lecteur du Code, il ne restera alors que le choix poignant entre l'un ou l'autre des deux contresens suivants : (a) affirmer qu'il y a trois différentes incriminations dans les articles 209 à 211 et aucune dans les trois articles suivants (alors que les six articles traitent de matières assez semblables); (b) conclure que chacun de ces six articles institue une incrimination spécifique, sans toutefois comprendre pourquoi les conduites visées par les trois premiers sont passibles d'une peine maximale de trois ans alors que celles dont il est question, dans les articles 212 à 214 , pourraient faire l'objet de peines d'emprisonnement allant jusqu'à sept ans (selon la règle de la peine générale instituée par l'article 951$)^{11}$. Pile ou face?

Il est probable qu'une partie du problème ici soit attribuable à l'emploi de l'expression «est criminellement responsable», que l'on trouve dans les six articles en question. On s'en sert, en effet, de manière peu systématique, voire abusive, dans la doctrine juridique, pour désigner tantôt la capacité, mesurée selon différents critères (âge, état mental), de commettre des crimes, tantôt la possibilité concrète (établie à partir des critères traditionnels de l'actus reus et de la mens rea) d'attribuer un crime à quelqu'un. En d'autres termes, on confond responsabilité criminelle et imputabilité. Des glissements de sens de cet ordre ne sont malheureusement pas rares dans le texte du Code. On s'imagine sans difficulté les problèmes d'interprétation qu'ils créent.

9. Art. 209 (devoir de fournir les choses nécessaires à la vie); art. 210 (devoir du chef de famille de pourvoir aux besoins des enfants); art. 211 (devoir des maitres envers leurs serviteurs).

10. Art. 212 (devoir des personnes qui font des opérations dangereuses); art. 213 (devoir des personnes en charge de choses dangereuses); art. 214 (devoir d'éviter des ornissions dangereuses).

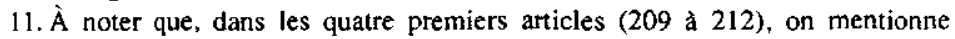
expressément la mort comme conséquence possible de l'omission; les deux derniers (213 et 214) ne font référence qu'au danger à la vie. 


\section{L'ARCHITECTURE DU CODE CRIMINEL CANADIEN DE 1892}

\subsection{Structure générale du Code}

Les 983 articles du Code criminel de 1892 peuvent être divisés en trois blocs distincts. Le premier bloc contient les dispositions dites introductives; le deuxième regroupe, entre autres, l'ensemble des énoncés d'incrimination; le troisième est consacré aux règles de procédure et de preuve. La distinction entre ces trois blocs, soulignons-le dès maintenant, se fonde moins sur un caractère absolu que sur un caractère dominant de différenciation.

Les 64 articles du bloc de dispositions introductives ne constituent pas une «partie générale » au sens que l'on attribue à ce terme dans les codes issus de la tradition du droit romain. À quelques exceptions près, ils n'énoncent pas des principes généraux devant guider l'interprétation et l'application des énoncés d'incrimination. Cette caractéristique constitue d'ailleurs le plus important des arguments dont on s'est toujours servi pour refuser à ce texte de loi l'appellation de code. Il serait, tout au plus, une consolidation de la common law ayant pour principales sources d'inspiration le Draft Code de Sir James Stephen (1878), les statuts britanniques en vigueur depuis la Conquête et les décisions des tribunaux. Quoi qu'il en soit, il serait erroné de conclure que de tels principes généraux n'existent pas en common law. Comme nous l'a déjà rappelé à juste titre la Commission de réforme du droit du Canada, ces principes se situent « tout simplement ailleurs que là où on (les) trouve dans un système codifié », soit dans les décisions jurisprudentielles ${ }^{12}$.

Le troisième bloc est constitué de 451 articles (articles 533 à 983) qui traitent, avec un rare luxe de détails, des rituels concernant, entre autres, les procédures applicables en matière d'accusations d'actes criminels et d'infractions punissables par voie sommaire, de règles de preuve ainsi que les procédures à suivre lors de l'exécution des sentences.

Les premier et troisième blocs sont, pour des raisons évidentes, de moindre intérêt pour les propos de cet article. Ce sont en effet les 468 dispositions contenues dans le deuxième bloc (articles 65 à 532) qui retiennent notre attention. Nous y trouvons, particulièrement, trois types d'articles: d'incrimination (proprement dits), de définition et de sanction.

Les premiers, au nombre de 366 , utilisent la forme courante d'incrimination décrite plus haut où la qualification pénale de l'acte ainsi que la peine qu'il entraîne sont énoncés en même temps. De ce total, 288 articles créent des actes criminels, 49 des infractions punissables par voie sommaire de culpabilité et 29 des infractions hybrides (actes criminels ou infractions

12. Op. cit., note 3, p. 22. 
sommaires). Cependant, plusieurs autres incriminations peuvent également être obtenues en combinant certains des 45 articles de définition aux 18 articles de sanction. Les 39 articles qui restent énoncent des règles diverses (d'interprétation, d'exclusion d'imputabilité, etc.) qui auraient d'ailleurs grand avantage à se retrouver dans l'un ou l'autre des deux autres blocs de dispositions.

Précisons cependant que la puissance «incriminatoire" du Code de 1892 (tout comme celle de n'importe quel autre code, common law et droit romain confondus) ne saurait en aucun cas être mesurée à partir de tels chiffres. Soyons plus précis: ce n'est pas parce que nous affirmons, par exemple, qu'il y a 288 articles qui incriminent des conduites dans le Code de 1892 qu'il faut conclure que l'on y trouve 288 incriminations. Le nombre réel d'incriminations instituées par ces articles dépasse largement ce chiffre. Des affirmations identiques peuvent être faites à l'égard des infractions punissables par voie sommaire de culpabilité et même de n'importe quel type d'incriminations obtenues par la combinaison d'articles de définition avec des articles de sanction. C'est que l'«article" - comme l'ont bien démontré Barberger et Lascoumes ${ }^{13}$ - constitue une unité de mesure tout à fait inadéquate du nombre d'incriminations, c'est-à-dire des "comportements autonomes sanctionnés ${ }^{14}$ " créées par une loi pénale. Un article peut en effet créer une ou plusieurs incriminations distinctes, indépendamment de l'appellation qu'il reçoit - et à laquelle tous les usagers du Code ont l'habitude de se référer - en note marginale. Les deux exemples suivants, tirés du Code de 1892 , illustrent bien cette importante distinction ${ }^{15}$.

238. Celui qui tente de se suicider est coupable d'un acte criminel et passible de deux ans d'emprisonnement.

124. Est coupable d'un acte criminel et passible de deux ans d'emprisonnement [celui qui prononce des paroles séditieuses], ou [publie un libelle séditieux], ou [prend part à une conspiration séditieuse]. (Les crochets sont de nous.)

L'article 238, identifié en note marginale par l'appellation «tentative de suicide $"$, ne crée de toute évidence que la seule incrimination que cette

13. Cf. Barberger, C. et P. Lascoumes, Le Temps perdu à la recherche du droit pénal, Paris, Ministère de la Justice, 1991, chap. I.

14. Ibid., p. 27.

15. À noter que c'est uniquement par souci d'économie d'espace que nous nous servons, dans cet exercice, d'articles concis. Il n'y a aucune corrélation à faire entre la longueur d'un article et le nombre d'incriminations qu'il crée. On peut instituer deux, trois, quatre incriminations en faisant preuve d'une grande sobriété verbale (même si cela est plutôt rare dans notre Code), et en créer juste une dans un article occupant deux pages entières du Code. 
appellation désigne. L'article 124, appelé «punition des actes séditieux », incrimine trois conduites distinctes : le fait de prononcer des paroles séditieuses, celui de les publier et, enfin, le fait de participer à une conspiration séditieuse. Ces trois conduites peuvent impliquer des acteurs distincts, dans des temps et des lieux distincts.

Le fait que l'on prenne ou non en compte cette distinction entre, d'une part, l'article (en tant que partie ou unité d'un texte légal dont le rôle est d'être le support matériel de différents messages du législateur) et, de l'autre, l'incrimination (en tant que forme particulière de qualification juridique de comportements) détermine différentes possibilités (niveaux) d'analyse des peines prévues dans un texte de loi criminelle. Les deux articles cités plus haut l'illustrent fort bien. Une étude des sanctions, fondée sur la seule notion d' «article», produirait le résultat suivant: les articles 124 et 238 du Code criminel de 1892 prescrivent, au total, deux peines d'emprisonnement de deux ans (une pour chaque article). Par contre, une analyse axée sur la notion d' «incrimination» identifierait, pour ces deux mêmes articles, quatre peines de deux ans d'emprisonnement (une pour chaque incrimination). Le premier niveau d'analyse nous apparaît suffisant pour dresser un tableau de l'appareillage répressif d'un code ou de n'importe quelle autre loi pénale considéré isolément. C'est celui que nous allons utiliser ici. Le deuxième niveau, qui met à contribution la notion d'incrimination, est le seul à notre avis en mesure de saisir adéquatement les mutations, ainsi que la nature des mutations, de la loi pénale pendant une période donnée.

\subsection{La structure des sanctions du Code de 1892}

L'article 6 du Code criminel canadien de 1892 énumère les punitions - c'est le terme qu'il emploie - auxquelles s'exposent les personnes reconnues coupables des infractions créées par cette loi. Outre les quatre peines " classiques » de cette fin de siècle - la mort, l'emprisonnement, le fouet et l'amende - on y trouve une liste de mesures diverses telles la destitution d'une charge sous la Couronne, la perte d'une pension ou allocation de retraite, l'incapacité d'exercer une charge publique, la perte du droit d'électeur, l'indemnisation des victimes de crimes contre la propriété, et nous en passons. D'autres mesures, non mentionnées dans cette liste - comme les confiscations, les saisies, l'incapacité de passer un contrat avec le gouvernement, entre autres - sont cependant prévues, ici et là, dans plusieurs autres articles du Code. L'article 6 passe égalemeñt sous silence les travaux forcés qui peuvent, du moins en théorie, aggraver les peines d'emprisonnement dans de nombreux cas. Cette conséquence supplémentaire de l'incarcération sera, dans certains cas, explicitement prévue par le Code et, dans 
d'autres cas, laissée à la discrétion du juge ${ }^{16}$. Les anciennes peines comme la mise hors la loi, la réclusion solitaire ou le pilori, de confiscation des effets mobiliers ayant entraîné la mort d'un être humain ( $\mathrm{sic}$ ) ainsi que l'arrêt de mort civile seront abolis ${ }^{17}$.

En matière d'infractions punissables par voie sommaire de culpabilité, les sanctions revêtent l'une des formes suivantes: amende seulement (environ un tiers des cas); emprisonnement seulement (figure plutôt rare); amende ou emprisonnement à défaut de paiement et, enfin, amende ou emprisonnement ou les deux, à la discrétion de la cour (figure typique). Les peines pécuniaires minimale et maximale sont de deux et mille piastres, mais on ne les trouve que dans de très rares cas. La valeur des amendes les plus fréquentes se situe plutôt entre cinquante et cent piastres. La longueur des peines d'emprisonnement, accompagnées (obligatoirement ou à la discrétion de la cour) de travaux forcés, varie entre trente jours et six mois. Ici encore, deux exceptions: une peine de huit jours, à l'article 477 et deux peines de douze mois aux articles 94 et 521 .

Le tableau des sanctions pour les actes criminels est autrement plus compliqué, sauf peut-être pour les peines d'amende, qui sont d'ailleurs très peu nombreuses: moins de quarante articles d'incrimination prescrivent une telle peine dont les montants, à quelques exceptions près, varient entre cent et mille piastres. Dans la grande majorité de ces cas, la peine pécuniaire s'ajoute à celle de privation de liberté. On laissera au juge, dans quelques articles, le choix entre l'une ou l'autre de ces sanctions, ou les deux simultanément. Autant dire que l'étalon de l'appareil punitif du Code de 1892, en matière d'actes criminels, est la peine d'emprisonnement. Nous lui consacrons la dernière section de cet article.

\subsection{La Reine, ses sujets et leurs biens : la peine d'emprisonnement dans le Code de 1892}

Il est encore trop tôt pour que l'on puisse définir la place exacte quj revient au Code criminel canadjen de 1892, par rapport aux législations pénales qui l'ont précédé, dans une échelle toute relative de modération. Des comparaisons entre les dispositions du Code et celles des ordonnances britanniques de 1763 puis 1774, pour le Bas-Canada, ainsi que celle de 1792, pour le Haut-Canada ${ }^{18}$, n'ont pas encore été menées de manière systématique. On ignore, pour l'instant, l'étendue de l'influence de deux

16. On trouve à l'article 955 du Code criminel de 1892 un certain nombre de règles générales concernant l'aggravation de l'emprisonnement par les travaux forcés.

17. Voir les articles 962 à 965 du Code criminel de 1892.

18. Voir, à ce sujet, (a) D. H. Brown, The Genesis of the Canadian Criminal Code of 1892, Toronto. The Osgoode Society, University of Toronto Press, 1986; (b) A. Morel, «La réception du droit criminel anglais au Québec ", Revue juridique Thémis, 1978, vol. 13, pp. 449-541. 
législations importantes de l'époque, entre autres, sur le Code de 1892 : les consolidations de lois criminelles de la Nouvelle-Écosse (1851) et du BasCanada $(1860),{ }^{19}$ en particulier de la première, que Brown ${ }^{20}$ considère comme une tentative novatrice en matière d'humanisation de la justice criminelle dans le territoire de l'Amérique du Nord britannique.

Quoi qu'il en soit, on ne prend pas grand risque en affirmant que, au moins sur un aspect, le Code de 1892 ne fait pas trop mauvaise figure à côté des législations pénales qui l'ont précédé. Nous faisons ici référence au recours à la peine capitale, qui se trouve limité, dans le Code de 1892, à six incriminations ${ }^{2 l}$. Certes, c'est encore très loin des trois seules peines capitales prévues dans la législation pénale de la Nouvelle-Écosse de $1851,{ }^{22}$ mais c'est sûrement mieux que les onze peines de mort qui étaient en vigueur au Canada au moment de la Confédération ${ }^{23}$.

Tableau 1

Peines d'emprisonnement, pour des actes criminels, prévues dans le Code criminel de 1892

Perpétuité -42
14 ans -53
10 ans -14
7 ans -62
5 ans -30
4 ans -1

3 ans -26

2 ans -68

moins de 2 ans -2

1 an -31

6 mois - 5

3 mois - 5

À la discrétiondu juge - 1

Total : 340 peines

En ce qui concerne le recours à l'emprisonnement, il est permis, même en l'absence de données complètes et systématiques pouvant servir de base de comparaison, d'affirmer qu'il est très important, aussi bien eu égard au nombre de cas prévu qu'à la sévérité des peines prescrites. Le tableau 1

19. Voir F. McIntyre, The Structure of Statutory Sentencing Provisions and the Development of Penal Law in Canada in the Middle of the Nineteenth Century: The Case of Nova Scotia and of Lower Canada (1851-1860), mémoire de maittrise inédit, Ottawa, Department of criminology, University of Ottawa, 1994.

20. Op. cit., note 18 (a).

21. Il s'agit de la trahison (art. 65), de l'aide à des sujets étrangers à entrer en guerre contre Sa Majesté (art. 68), de la piraterie (art. 127), de la piraterie avec violence (art. 129), du meurtre (art. 231) et du viol (art. 267). Soulignons que la peine de mort est incontoumable pour les cinq premières infractions ci-dessus. Dans le cas du viol, le Code donne au juge le choix entre cette peine et l'emprisonnement à vie.

22. Op. cit. note 19, p. 52 .

23. Ibid., p. 64 . 
donne un premier aperçu de la situation. On constate, à sa lecture, que même si la peine la plus fréquente - appelons-la la peine modale -- est de deux ans d'emprisonnement ( $20 \%$ du total), la balance de la sévérité penche nettement du côté des peines égales ou supérieures à cinq ans : on y trouve pratiquement $60 \%$ des peines d'emprisonnement prévues par le Code de 1892. Réunies, les peines d'emprisonnement à vie et de 14 ans d'incarcération comptent pour presque la moitié d'entre elles.

Le tableau 2 montre en détail la distribution de ces peines d'emprisonnement parmi les cinq grandes familles de biens juridiques protégés par le Code : l'ordre public; l'administration de la loi et de la justice ; la religion, les mours et la commodité du public; la personne et la réputation et, enfin, la propriété, les droits résultant des contrats et les actes de commerce. À chacune de ces familles, le législateur a consacré, dans cet ordre, un titre du Code. Cette disposition des matières traitées - de Sa Majesté aux opérations de commerce - où le caractère public du bien juridique protégé s'affaiblit graduellement, est encore visible dans le Code actuel malgré l'état de délabrement dans lequel on l'a laissée après un siècle de réformes. C'est d'ailleurs en suivant une logique semblable que Blackstone abordera les grandes familles d'infractions dans ses Commentaries, publiés plus d'un siècle auparavant. ${ }^{24}$

Le tableau 2, comme on peut le constater, fait également état des six peines capitales prescrites par le Code. La décision de les inclure, à côté des sanctions de privation de liberté, se justifie par le fait que l'adoption par le législateur de la peine capitale signifie, entre autres, son aveu de l'insuffisance punitive de l'emprisonnement à vie. À défaut de pouvoir établir, entre ces deux peines, un écart mesurable, on peut y voir une continuité logique dans une échelle sans plafond de l'action répressive.

L'identification de la peine modale, pour les différentes familles de biens juridiques protégés figurant au tableau 2, donne déjà une idée assez précise de l'importance relative que leur attribue le législateur de l'époque. Les peines modales sont donc les suivantes:

- crimes contre l'ordre public : emprisonnement à vie ;

— crimes contre l'administration de la loi et de la justice : deux ans;

24. Dans l'ordre proposé par Blackstone, les crimes contre Dieu et la religion trônent en haut de l'échelle d'importance des biens juridiques protégés. Il est question ici, pour le juriste anglais, de l'apostasie, de l'herésie, du blasphème, des jurons, de la sorcellerie et de l'impudicité, entre bien d'autres. C'est bien la foi et ses symboles qui sont mis sous la tutelle du droit pénal. Voir W. Blackstone, Commentaries on the Laws of England, vol. IV : Of Public Wrongs, Oxford, Clarendon Press, 1769 (réimprimé en 1966 par Wildy \& Sons Ltd., Londres). Dans le Code de 1892, on ne trouve que quatre infractions en cette matière, une sur le libelle blasphématoire et trois concernant les entraves aux pratiques religieuses. C'est moins la foi que la liberté de culte qui se trouve ici protégée. 
Tableau 2

Peines de mort et d'emprisonnement dans le Code criminel de 1892

\section{Peines}

\section{Crimes contre...}

\begin{tabular}{|c|c|c|c|c|c|c|c|c|c|c|c|c|c|c|c|}
\hline & Mort & Perp. & 14 ans & 10 ans & 7 ans & 5 ans & 4 ans & 3 ans & 2 ans & $<2$ ans & 1 an & 6 mois & 3 mois & Disc. & Total \\
\hline l'Ordre public & 4 & 9 & 3 & 1 & 5 & 1 & 0 & 1 & 6 & 0 & 7 & 2 & 1 & 1 & 41 \\
\hline la Loi et la Justice & 0 & 1 & 4 & 2 & 7 & 3 & 0 & 0 & 12 & 0 & 4 & 1 & 1 & 0 & 35 \\
\hline la Religion/les Mœeurs & 0 & 1 & 2 & 2 & 0 & 4 & 1 & 0 & 11 & 0 & 8 & 1 & 0 & 0 & 30 \\
\hline la Personne & 2 & 12 & 6 & 1 & 12 & 5 & 0 & 8 & 10 & 2 & 3 & 0 & 0 & 0 & 61 \\
\hline la Propriété & 0 & 19 & 38 & 8 & 38 & 17 & 0 & 17 & 29 & 0 & 9 & 1 & 3 & 0 & 179 \\
\hline Total : & 6 & 42 & 53 & 14 & 62 & 30 & 1 & 26 & 68 & 2 & 31 & 5 & 5 & 1 & 346 \\
\hline
\end{tabular}


- crimes contre la religion et les mœurs : deux ans;

- crimes contre la personne: sept ans et emprisonnement à vie (ex aquo);

- crimes contre la propriété : sept et quatorze ans (ex aquo).

Ce simple indicateur met déjà en relief trois biens juridiques plus (farouchement) protégés que les autres: l'ordre public, la personne et la propriété. Ce n'est manifestement pas de puritanisme victorien que se nourrit ce Code.

On pourrait également, en faisant appel à un expédient quantitatif fort rudimentaire et sans doute pour cela très efficace, mettre davantage en évidence cette tendance. Il s'agit tout simplement d'un indicateur, auquel on peut accoler l'appellation ronflante d' «indice de sévérité des peines". que l'on obtient en mettant en rapport le haut (la somme des fréquences des sanctions les plus sévères) avec le bas (la somme des fréquences des sanctions les moins sévères) de l'échelle des peines pour chaque bien juridique protégé. Comme toute entreprise de mesure, celle-ci part d'un choix arbitraire. Voici le nôtre : nous considérons plus sévères les peines égales ou supérieures à cinq ans et moins sévères celles égales ou inférieures à trois ans. La seule peine de quatre ans figurant au tableau (erreur des réviseurs du Code ou dilemme du législateur?) sera exclue du compte. Quoi qu'il en soit, cet incident a au moins l'avantage, non négligeable en l'occurrence, de tracer une frontière imaginaire entre deux zones de l'échelle de sévérité du Code. Pour des raisons évidentes, la peine que le législateur a laissée à la discrétion du juge (article 73), qui figure à l'extrême droite du tableau, ne sera comptée nulle part.

À partir de ces critères, on peut attribuer l'indice de sévérité des peines suivant, par ordre croissant de grandeur, à chaque famille de biens juridiques protégés :

- la religion et les mœurs $(9 / 20)$;

- l'administration de la loi et de la justice (17/18);

- l'ordre public $(23 / 17)$;

- la personne et la réputation (38/23);

- la propriété, les contrats et les actes de commerce (120/59).

En d'autres termes, le total des peines moins sévères, en matière d'infractions contre la religion et les mœurs (20), est deux fois plus grand que celui des peines plus sévères (9), exactement l'inverse de ce qui se produit dans le domaine des crimes contre la propriété (59 peines moins sévères contre 120 plus sévères).

En effet, $60 \%$ des peines plus sévères se retrouvent dans la catégorie des crimes contre la propriété. À la rescousse de ce bien juridique, on fera appel à la presque moitié de peines d'emprisonnement à vie et aux trois 
quarts environ des peines de 14 ans de réclusion prescrites par le Code. Et on court sérieusement le risque de ne pas apprendre grand-chose à personne en étalant la liste des transgressions spécifiques auxquelles ces sanctions s'adressent. Contentons-nous plutôt de dire que pour les modalités moins plébéiennes de ces illégalismes (fraudes, escroqueries, infractions diverses relatives au commerce), le nombre de peines moins sévères est pratiquement trois fois plus grand que celui des peines plus sévères. Ce qui, faute de mieux, pourra bien être la bonne nouvelle que, tradition oblige, on se doit d'apporter au bout d'un long voyage.

\section{CONCLUSION}

Les images fragmentaires du Code criminel de 1892, présentées dans cet article, ne constituent bien entendu que les résultats d'une première étape d'approche d'une vaste entreprise législative commencée en 1892. Encore faut-il qu'on le saisisse, ce Code, non pas seulement en tant qu'expression écrite et finale de la volonté du législateur, ou simple souvenir des intransigeances d'une époque, mais comme totalité en mouvance permanente dans l'espace d'un siècle. En ce sens, le Code n'a pas de date, il est intemporel autant qu'il demeure l'expression la plus achevée d'une rationalité particulière : celle qui revendique une nature à part à un certain nombre de rapports conflictuels en leur prescrivant une forme de prise en charge (la sanction pénale) qui en exclut toute autre. C'est sur cette toile de fond théorique que devra se poursuivre l'analyse des différentes étapes de sa construction.

En attendant de pouvoir se hisser à de tels sommets, il nous semble que l'exercice auquel nous nous sommes livré ici, ainsi que les premières tentatives de le prolonger au-delà de $1892^{25}$, ouvrent plusieurs pistes de réflexion. Nous nous bornerons à indiquer brièvement une seule d'entre elles, poussé sans doute par la conviction qu'elle interroge un thème d'une grande actualité : celui des mouvements, d'élargissement et de rétrécissement, du pouvoir de régulation de l'État et, par conséquent, des moyens concrets d'intervention qu'il déploie pour assurer ce pouvoir.

De la vaste entreprise conceptuelle à laquelle les débats autour de ce thème ont donné lieu ${ }^{26}$, deux notions - criminalisation et pénalisation -

25. Les versions de 1927 et de 1955 du Code criminel ont déjà fait l'objet d'un certain nombre d'analyses semblables à celles qui ont été développées dans cet article. bien que moins approfondies. Il serait impossible, faute d'espace, d'en faire état ici.

26. On trouve dans les travaux de Kerchove une liste imposante de ces concepts, particulièrement pour la série en "dé" : déjuridiciarisation, délégalisation, déjuridictionnalisation, déjusticialisation, et nous en passons. Voir, entre autres, $M$. van de Kerchove, "Les différentes formes de baisse de la pression juridique et leurs principaux enjeux ", Cahiers de recherche sociologique, automne 1989, vol. 13, pp. 11-29. 
ainsi que leurs contraires - décriminalisation et dépénalisation — ont été particulièrement retenus dans les discours des criminologues, chercheurs autant que praticiens. La somme des interventions faites en cette matière, dont nous ne pouvons évidemment pas faire ici le bilan, tend à indiquer que l'on réserve le plus souvent l'utilisation de ces termes aux épisodes flagrants, incontournables, d'émergence ou de disparition d'une incrimination, d'augmentation ou de diminution de la sévérité d'une peine. Un exemple parmi bien d'autres : on dira que le Bill omnibus a décriminalisé l'homosexualité et l'avortement (ce dernier sous certaines conditions), dans la mesure où cet acte du Parlement a rayé du Code, ou modifié substantiellement, les articles qui incriminaient ces conduites. Nous sommes de plus en plus porté à croire que de tels épisodes ne constituent qu'une des formes de modification des dispositions législatives en matière pénale. Il y en a d'autres, beaucoup plus subtiles, souvent insidieuses, pas moins efficaces pour autant.

S'il y a bien quelque chose qu'une lecture de deux versions, même relativement rapprochées, du Code nous apprend, c'est qu'il est possible de criminaliser davantage sans introduire aucune nouvelle incrimination dans le Code ou, au contraire, de faire reculer le processus de criminalisation sans que le nombre d'incriminations diminue. Voici, entre autres, trois scénarios qui illustrent ces situations.

(i) En modifiant (parfois légèrement) la đéfinition d'un acte ou, plus directement, les termes par lesquels on énonce sa qualification pénale, on peut fort bien soit élargir, soit rétrécir l'espace événementiel couvert par l'incrimination - l'infraction reste, mais elle n'est plus la même, elle n'autorise plus la même lecture de la réalité. Les acteurs sociaux qui évoluent dans l'espace redéfini ne sont plus les mêmes, les actions incriminables non plus. Les articles du Code criminel qui affichent des listes interminables de verbes d'action sont des terrains fertiles pour ce genre de mutations de la portée de la loi pénale.

(ii) On peut obtenir des résultats semblables en modifiant, cette fois-ci, des règles d'exclusion d'imputabilité (immunités accordées par la loi à certaines catégories de personnes, par exemple) ou d'exclusion de responsabilité (modifications des limites d'âge). Elles sont d'ailleurs assez nombreuses dans le Code, et pas toujours en évidence. Dans bien des cas, on les retrouve incluses dans l'énoncé d'une incrimination.

(iii) À la limite, il est plausible que l'on produise des effets tout aussi importants, sinon plus, en matière de criminalisation et de décriminalisation en apportant des modifications aux règles de procédure et, particulièrement, de preuve. On parle ici, bien entendu, d'un effet de l'action conjuguée du législateur (sur le texte de la loi) et du juge (au plan de l'interprétation 
jurisprudentielle). Compte tenu de l'état actuel d'avancement de nos recherches, cette modalité demeure pour l'instant hypothétique.

En ce qui concerne, finalement, la pénalisation et la dépénalisation, inutile d'insister sur le fait que leur mouvance se trouve en partie à la remorque des modifications des balises de la criminalisation. En principe, la suppression totale ou, au contraire, la création d'une incrimination implique un mouvement de recul ou de progression, respectivement, de l'emprise «sanctionnatrice» de l'État. Cela n'exclut pas, cependant, que l'on puisse identifier des modalités spécifiques de pénalisation et de dépénalisation qui violent de manière flagrante la règle que nous venons d'énoncer. Le cas d'espèce à évoquer ici est le suivant: le retrait d'une incrimination (on parle donc de décriminalisation au sens propre du terme) n'élimine pas la possibilité que certaines conduites qu'elle visait soient prises en charge soit par une incrimination connexe, soit par une incrimination dont l'espace événementiel aurait subi des modifications. On parlera alors de pénalisation ou de dépénalisation pour cette conduite spécifique, dans la mesure où la nouvelle règle qui l'incrimine prescrit des sanctions plus ou moins sévères que celles dont elle était passible auparavant.

Un constat à retirer de ces propos? Probablement celui de la totale inutilité de prétendre s'attaquer à ce pouvoir — pouvoir de discrimination, de stigmatisation, de bannissement - sans préalablement mettre à nu la fragilité de son discours. Car ce qui frappe le lecteur du Code, ce n'est pas tant ce qu'il dit, mais plutôt tout ce que l'on est capable de lui faire dire. Il est même fort possible que l'on puisse voir, dans ce trait, non seulement les limites des codes d'hier et d'aujourd'hui, mais de tout Code criminel, en tout temps, et de ceux en particulier qu'on ne cesse de nous promettre pour demain. 